\title{
Capteurs ou Senseurs et la théorie du A QUOI ?
}

\author{
Pierre BONTON \\ Courriel : Pierre.Bonton@univ-bpclermont.fr \\ Université Blaise Pascal - Département de physique -Institut Pascal - UMR 6602 CNRS 63177 Aubière
}

RESUME : Un senseur au sens évaluer les sensations peut-il remplacer un capteur au sens donner une valeur juste de la mesure. Le capteur a besoin d'être calibré, le senseur ne s'intéresse qu'au quoi de l'objet. Cinq membres d'une même société s'interrogent sur l'évolution du capteur vers le senseur. Chacun a une spécialité soit sur le savoir, sur le savoir faire, sur le savoir faire faire, sur le savoir être ou sur le faire savoir. L'histoire se passe sur 20 ans à partir de 2013 ...

Mots clés : capteur, senseur, théâtre et sciences, connaissances, compétences

\section{INTRODUCTION :}

Nous sommes en 2013, l'histoire commence tout au début de la chaine d'acquisition de données; la question est: mon capteur est-il bien calibré ? Cinq protagonistes :

- Madame Marie CREUDUI (MC) 50 ans, elle détient, ou croit détenir, les connaissances, le savoir. Elle est assez libre et émancipée pour prendre et faire ce qu'elle désire. Elle est à la tête du service Recherche et Développement.

- Monsieur Sorggee FOGBOSSAU (SF) 30 ans, est fier de ses compétences qu'il a acquises au cours de sa formation. Il est formé, normé et utile dans un monde socio-économique dont il est le rouage, il Sait Faire ...

- Monsieur Serge-Francis FEUXDASP (SFF) 48 ans, est le chef d'équipe. Les relations avec MC sont assez tendues, c'est lui qui Sait Faire Faire.

- Madame Sylvie EDREPERAU (SE) 28 ans, dirige la gestion des ressource humaines. Elle déplace les pions en fonction des besoins de l'entreprise. Pour elle l'homme est une ressource et elle le gère en tant que tel, elle connaît le Savoir Etre !

- Monsieur Fabrice SOBBRE (FS) 37 ans, est le commercial. Il doit faire du chiffre. Il est jovial, rougeaud, manipulateur, sympathique et écrasant les autres. Sa formation à l'ESC l'a étalonné pour ça, il Fait Savoir.

Tous ces gentils membres appartiennent à la même entreprise qui fabrique des capteurs en relation étroite avec un laboratoire de recherche d'une université provinciale néanmoins «labexisée » autrement dit Laboratoire d'excellence (LABEX).

\section{Synopsis :}

Pourquoi ne pas remplacer les capteurs par des senseurs ? Telle est la question qu'un chercheur, le professeur ZALEIG, se pose à la fin du $20^{\mathrm{ème}}$ siècle. Un capteur qu'il soit passif (thermomètre, caméra ...) ou actif (sonars, télémètre ...), doit être modélisé et calibré.

Modéliser c'est trouver une équation qui le représente, généralement une équation différentielle ! Fi des mots !
Prenons une transposition: Modéliser c'est, par exemple, réaliser le patron d'un vêtement. Calibrer c'est prendre les mesures d'une personne pour adapter le modèle (patron) à la personne. Un modèle reste un modèle avec ses défauts même si c'est un top modèle ! Les mesures ont une marge d'erreurs, elles sont incertaines. Modéliser un capteur et le calibrer sont deux opérations qui peuvent être complexes. De plus un capteur actif peut perturber et modifier le monde qui l'entoure.

A la même époque d'autres chercheurs travaillent sur l'incertain. Ce sont des théories que l'on nomme «la logique floue », «l'évidence» ou encore la théorie de Dempster-Shafer. La question est de savoir, si à partir de données incertaines et d'algorithmes flous, une machine peut prendre une décision?

Voilà donc que notre chercheur franchit le Rubicon. Il se demande: Pourquoi ne pas remplacer les capteurs par des senseurs ; c'est-à-dire plus de mesure mais des sensations comme nos cinq sens, comme la nature, comme un coup de crayon pour faire l'esquisse d'un vêtement. Et, se dit-il : cela doit suffire et donc finis les modèles, finies les mesures !

Après 25 ans de recherche, l'idée a fait son chemin jusqu'à intéresser une entreprise qui fabrique des capteurs.

Notre chercheur vit dans un monde citoyen. Et, souvent, il y a implication de l'histoire sur les sciences et réciproquement. Durant ces 25 années, les mentalités ont aussi évolué en même temps que les recherches du professeur ZALEIG. Nous ne nous intéressons plus au modèle (le Pourquoi) ni aux mesures (le Comment) encore moins aux contenus (le Quoi) mais seulement aux «A QUOI » ça sert ? (la boite noire). La méthode des essais/erreurs est reine!

La pièce imaginée que je vous propose (toute ressemblance est fortuite... encore que !) débute 25 ans après les premiers travaux de notre chercheur. La théorie du «A QUOI » est déjà bien présente et les capteurs ont du souci à se faire.

L'histoire se passe sur 20 ans, elle débute en mars 2013 dans une entreprise qui fabrique des capteurs. Notre chercheur a pris sa retraite et il est quelque peu oublié (sans doute pas assez de publications !). 


\section{ACTE 1 (21 mars 2013)}

\subsection{Scène 1 : devant la machine à café :}

Marie : Salut Sorggee, ça roule... tiens il y a longtemps que je voulais te dire. Nous sortons de la même école d'ingénieurs et pourtant nous ne raisonnons pas pareil ?

Sorggee : Ah oui madame C (prononcé si), il y a eu la loi Savary qui est passé par là. A l'université on n'enseigne plus, on forme maintenant. Les UEF se sont transformées en UFR depuis 1984. Et en plus depuis 2011 on ne dispense plus des savoirs mais des compétences, c'est plus utile pour le monde socioéconomique. Il faut vivre avec son temps.

Marie : Ouais ! Je pense différemment. Pourtant nous avions les mêmes profs ou à peu près...même si on a 15 ans d'écart?

Sorggee : 20

Marie : Hum..

Sorggee : Et oui il faut bien calibrer nos différences... Tiens, tu vois Madame $\mathrm{C}$ pour bien calibrer il faut un bon étalon, c'est toi qui le répète...

Marie : Oui ça va, et tu te crois drôle?

Sorggee : Drôle, non! Ah oui, je veux dire étalonner par rapport à une référence. Dans notre cas c'est le temps $15-20 \ldots$ tu vois !

Marie : [1] [2] Non le temps n'est pas une référence, mais une variable. C'est un simple paramètre permettant de relier entre eux les phénomènes physiques dirait Galilée. Mais les choses se compliquent : d'absolu il passe à relatif avec l'irruption de la relativité restreinte. Tu connais Albert? La référence c'est la durée qui se définit dans le même espace, la même vitesse. Bref, on aura toujours 20 ans d'écart, quel que soit le temps. Et ça, c'est invariable, 20 ans d'écart. Le problème est de trouver quelque chose d'invariable ou alors de peu variable...

Serge-Francis: Hep vous deux, vous avez fini de monopoliser la machine?

Marie : On philosophe...

Serge-Francis : Ici pas de place pour la philosophie, on travaille; retournez à vos études.

Et vous Sorggee, allez calibrer.

Sorggee : Justement j'ai un problème avec le dernier capteur conçu au Labex, il diffère de la référence...

Serge-Francis : Enfin avec Matlab, la méthode des moindres carrés, il ne peut que suivre la référence et non dériver?

Sorggee : Je ne comprends pas ce que vous dites. Ma feuille de route est claire, je branche, j'appuie, j'attends. Si la lumière est verte, je valide dans le fichier Excel. Ce sont mes compétences et en haut c'est ce qu'ils désirent, des chiffres, des camemberts....

Serge-Francis : Très bien et alors ?

Sorggee : Tous les derniers capteurs virent au rouge et mes camemberts ont la même couleur !

Marie : Le modèle ...
Serge-Francis : Oh toi ça va, je t'ai dit de retourner à tes études.

Marie : Tchao. Tiens voilà le top modèle !

Sylvie : Bonjour tout le monde, ça va! Un problème ?

Serge-Francis : Non, les nouveaux capteurs virent au rouge, mais j'ai la solution. On va diminuer la précision. On a de la marge sur les incertitudes. Allez Sorggee, je vous ai donné la solution au boulot et « écologissez » vos camemberts ah ah ah...

2.2 Scène 2 : toujours devant la machine à café. Sylvie seule avec Serge-Francis :

Sylvie: En parlant de précision, notre machine d'étalonnage est au point maintenant, Sorggee est très compétent, mais je pense que nous pouvons automatiser le test, les camemberts se rempliront automatiquement. Il suffit de régler les incertitudes comme vous le soulignez si justement.

Je pense utiliser les compétences de Sorggee au contrôle qualité. Qu'en pensez-vous ?

Serge-Francis : Il faudrait en discuter avec lui ?

Sylvie : C'est vous le chef, je vous demande votre avis?

Serge-Francis : Ce n'est pas impossible ...

Sylvie : Ok, c'est fait, la demande de changement de poste est partie hier. Les horaires de travail sont un peu différents, mais il s'adaptera ...

Au fait Marie n'est pas encore à la retraite ?

Serge-Francis : Elle a presque mon âge, nous avons suivi les mêmes études et ...

Sylvie : Tiens voila notre cher commercial...

Salut Fabrice comment vas-tu ?

Fabrice : Salut toi. Tu n'es pas venue hier au soir avec notre client Russe. Trop super, c'est un vrai étalon question vodka! Sur les treize testées, trois ont obtenu le label vert. Bref, tiens, il t'a réclamée ! Mais, même sans toi, je l'ai dans la poche. Il a signé le contrat sur les nouveaux capteurs. Nous avons un intervalle de confiance au beau fixe avec lui et ça grâce à moi.

Sylvie: Bon ça va, mais heureusement que SergeFrancis trouve des solutions.

Serge-Francis : J'ai résolu un problème de calibrage.

Fabrice : Bravo. On mange ensemble. Tu m'expliqueras ça pour mes arguments commerciaux. J'ai croisé Marie, pas encore à la retraite ?...

Serge-Francis : Non (il sort).

2.3 Scène 3 : toujours devant la machine à café. Sylvie seule avec Fabrice :

Fabrice: $\mathrm{Au}$ fait, je suis allé faire une formation technique comme tu me l'avais conseillée. Je peux te dire que j'ai acquis des compétences.

Sylvie : Ah oui, sur quoi ?

Fabrice : Sur la théorie de la croyance.

Sylvie : De quoi ? 
Fabrice : De la croyance ; en fait, nous n'aurions plus besoin de capteurs fiables. Il suffit qu'ils donnent de l'incertain. Et, c'est les sensations qui feront le reste. Tu sais « je sentis avant de penser » disait Rousseau.

Sylvie : Rousseau?

Fabrice : Et bien .... Heu... bref. Les Anglo-Saxons nomment les capteurs «sensors ». Tiens, toi tu ressens, tu ne mesures pas. Et, ça ne t'empêche pas de décider. Le verbe croire, il a toutes les significations du « credo » jusqu'au «peut-être », étonnant non? Cette théorie s'appelle non seulement de la croyance mais aussi de l'évidence. Tu vois c'est du flou ! D'ailleurs tout ça est basé sur la théorie de la «logique floue » [3]. Tu te rends compte associer le certain de la logique avec le flou.

Sylvie : Je ne comprends rien...

Fabrice : Moi non plus, enfin pas tout. Mais ce que je vais proposer à notre prochaine réunion «AvenirActions », c'est de supprimer carrément le poste « calibrage » qui pose problème et de le remplacer par le logiciel de danseur-chauffeur ou de senteur-chatteur enfin je ne sais plus... Bref, le logiciel qui donne du sens à l'incertain grâce à des multi-capteurs flous.

Sylvie : Je comprends de moins en moins. Tu en as parlé au Labex et à Marie ?

Fabrice : Non, elle ne comprend rien au monde socioindustriel. Ils ont du mal avec les compétences et ....

(Madame connaissance passe)

Marie : Encore devant la machine à café, à discuter !

Fabrice : Bon ça va, on discutait de choses concrètes sur la logique floue.

Marie : Ah oui ! C'est une théorie déjà ancienne développée par Arthur P Dempster et Glenn Shafer, et aussi Didier Dubois et Henri Prade [4] à Toulouse dans les années 60-70. C'est pas mal et ça conforte la mesure pour prendre une décision quand les capteurs donnent des mesures incertaines. Mais, naturellement ça ne peut pas remplacer une mesure fiable quand elle peut être réalisée. D'où l'intérêt d'avoir un bon modèle pour calibrer.

Fabrice : Ce n'est pas ce que disent les experts de mon dernier stage technique.

Marie : De ton premier tu veux dire !

Sylvie : Bon ça va, c'est quand même grâce à Fabrice que nous remportons des marchés à l'exportation, en particulier avec les Russes. Et en tant que responsable de la GRH, je...

Marie : Ah oui ! La GRH, ça me fait penser à gestion de la production porcine dans le bas limousin. Une femme, un homme ne sont pas des ressources comme une machine et ne se gèrent pas comme un compte en banque. Je préfère les mots comme Relations Humaines.

Sylvie : Ca va, on connait votre chanson, la gestion des ressources humaines remplace le service du personnel et c'est tant mieux et plus efficace.

Marie : Tu devrais ouvrir tes yeux et tes oreilles et non capter seulement le son des sirènes du CAC 40.
Tiens, au fait, Fabrice ça a duré combien de temps ton stage technique?

Fabrice : Une journée pourquoi?

Marie : Pour rien! Bon je vais voir Sorggee, il doit changer son modèle pour pouvoir calibrer au mieux les capteurs que nous fabriquons. D'ailleurs, je me demande si une machine ne pourrait pas le remplacer ... Remplacer les compétences par une machine !

Heu, vous êtes très compétents vous?

Fabrice : Ne t'inquiète pas. Bientôt on remplacera les capteurs par des senseurs. Et toi pas sûr que tu aies ta place !

(Marie sort en souriant)

Fabrice : Elle m'énerve, je me demande quand notre PDG va la virer?

Sylvie : Ca ne risque pas. Au dernier conseil, il l'a défendue et il veut même développer son service.

Fabrice : Elle couche?

Sylvie : Tu veux dire elle a couché... Hi Hi Hi ...

\subsection{Scène 4 : Marie va voir Sorggee dans son atelier de mesures.}

Marie : Tu devrais te méfier de Sylvie, elle n'a aucun esprit critique. Elle ne comprend pas que plus tu augmentes l'imprécision, plus tu diminues la confiance. Je pense qu'elle veut automatiser ton poste. Je sais, elle en a parlé au Labex.

Sorggee: $\mathrm{Tu}$ crois que la compétence peut être remplacée par une machine, un algorithme ?

Marie : Oui et lorsque l'on commence par déformer les commerciaux avec la théorie de Dempster/Shafer, les senseurs ne sont pas loin.

Sorggee : Quoi un censeur ?

Marie : Non des senseurs. Enfin quoi, un capteur mesure une valeur la plus juste possible. Un senseur mesure les sensations. C'est un anglicisme de « sensor» déformé.

Sorggee : Ah oui ?

Marie : Un peu comme toi, tu sens le chaud voire le brûlé sans le mesurer, tu goutes sans connaître les teneurs en composants naturels ou chimiques, tu entends sans savoir le nombre de décibels, tu mates sans retenue et en plus tu fais jouer ton instinct.

Entre les senseurs flous, la théorie de la croyance et l'intelligence artificielle, voilà les nouvelles machines. C'est ce qu'ils étudient au Labex. Tu piges?

Sorggee : Oui enfin, pour moi un capteur, comme tu l'as dit, doit donner une mesure la plus juste possible quelque soit l'environnement. Alors pour les sensations on va attendre encore non?

Marie : Pas si sûr, les capteurs qui sont actifs on commence à en revenir. Tu comprends, ils perturbent l'objet. Quand tu envoies un rayonnement par exemple un laser pour mesurer une distance du capteur à l'objet, l'onde se réfléchit sur l'objet et peut le perturber.

Sorggee : Ah oui, c'est le principe du télémètre laser. On le fabrique et bien calibré, c'est mon job, il donne 
des mesures précises et utiles. Parles-en aux Russes ? Nous n'avons jamais eu de retour ni pour mauvaises mesures et ni pour dégradation de l'objet.

Marie : Oui. Encore faut-il faire attention à la longueur d'onde et à la puissance du laser, sinon il est dangereux pour l'homme!

Tiens, un autre exemple avec les sonars. Il y en a partout pour étudier les fonds marins et aussi pour connaître les mouvements des animaux marins. Et bien, une étude a montré que les ondes des sonars perturbaient la vie sous-marine en particulier des baleines, j'avais lu ça dans Libé il y a quelques temps [5], mais je n'avais pas poussé plus loin. Et actuellement il y a une grosse polémique entre l'armée US et les chercheurs à propos du LFAS (Low Frequency Active Sonar) construit par l'US Navy. La revue Nature a publié un article sur ce sujet en juin 2012 [6].

Alors, tu vois; ce n'est pas si idiot d'étudier des senseurs passifs qui donnent des sensations et d'associer leurs mesures incertaines à des algorithmes provenant de la logique floue pour prendre une décision.

Sorggee : Putain, tu as de la chance dans dix ans la retraite...

Marie : Vingt tu veux dire...

\section{ACTE 2 (20 ans plus tard)}

(Tout le monde était là, ou presque, pour arroser le départ à la retraite de Marie)

Le lendemain 22 mars 2033.

\subsection{Scène 1 : Sylvie et Serge-Francis devant la} machine à café, puis Fabrice.

Sylvie : Enfin ...

Serge-Francis: Oui, elle devenait de plus en plus pénible. Les réunions techniques vont être plus calmes et plus courtes.

Sylvie : Oui.

Serge-Francis : Au fait tu as des nouvelles de SF enfin de Sorggee?

Sylvie : Oui, il est toujours en dépression, ça fait maintenant plus de cinq ans, non?

Serge-Francis : Peut-être... Tiens voila Fabrice, enfin je veux dire Monsieur le Directeur maintenant.

Fabrice : Salut vous deux. Avec le nouveau PDG, on a enfin reconnu mes compétences. C'est moi il y a vingt ans qui a proposé la théorie Dempster-Shafer et les senseurs.

Serge-Francis : Oui, mais c'est Marie qui l'a implantée, un peu contrainte d'ailleurs et sous pression du Labex qui était lui-même sous pression des publications...

Fabrice : Oui ça va. L'important ce n'est pas de savoir implanter mais de dire à quoi ça sert. J'espère que tu connais la théorie du A Quoi ? Je viens de faire un stage là dessus...

Serge-Francis : Non. C'est quoi ?

Fabrice: Le gars nous a expliqué en remontant jusqu'aux Grecs. Vous allez comprendre. Enfin je vais vous dire ce que j'ai retenu.

Le titre du stage était : «La civilisation du A Quoi ». En gros: les philosophes grecs s'intéressaient au «pourquoi » des choses. J'appellerai ça, la connaissance du questionnement. Plus tard, à partir du $17^{\text {ème }}$ siècle, les philosophes, qui sont aussi pour la plupart des scientifiques, développent la pensée du « comment » et ceci jusqu'au début du $21^{\text {ème }}$ siècle. Le «pourquoi » et le «comment» sont, sans doute, les bases de la connaissance.

D'après notre conférencier, aujourd'hui en 2033 les gens ne s'intéressent plus qu'aux «à quoi ». 90\% des citoyens consomment du «à quoi » uniquement. Les autres sont au service et esclaves du «A QUOI».

Sylvie : Mais qu'est-ce-que ça à voir avec nous. Tout ça, c'est de la masturbation intellectuelle à mon avis ?

Fabrice : Pas tant que ça. Et puis la masturbation intellectuelle ou non est préconisée par les psys et le monde médical ...

Sylvie: Bon ça va...

Fabrice : Tu n'es pas drôle toi aujourd'hui ? Bref ! Nous avons eu une discussion ensuite sur justement la théorie du «à quoi ».

Serge-Francis : Ah bon ça m'intéresse, explique.

Fabrice : Le «à quoi », n'est pas le contenu mais le à quoi ça sert. Le reste de savoir comment ça marche et de pourquoi on l'utilise. On s'en fout.

Le «A Quoi » s'appuie sur deux démarches : une bien connue: les essais/erreurs. Il suffit de regarder les enfants jouer mais c'est aussi une méthode scientifique que beaucoup de chercheurs utilisent.

Serge-Francis : C'est vrai, je l'applique régulièrement, jusqu'à ce que ça marche. J'en ai quelques fois marre de chercher à comprendre.

Fabrice : Comme moi ! La deuxième méthode est la «logique floue ».

Serge-Francis : Je comprends bien la logique classique et numérique, elle est basée sur toutes les combinaisons possibles de 0 et de 1 . Un état correspond à une combinaison précise. Tiens j'ai un exemple le «bibi binaire » de Bobby Lapointe [7] qui a été repris dans un spectacle de François Fabre [8] que j'ai vu il y a longtemps en 2010 ou 2012 je ne sais plus....

Fabrice : je ne connais pas.

Sylvie : Moi non plus

Serge-Francis : Pourtant vous devriez écouter c'est super drôle et explicatif...

Fabrice : OK, peu importe, je continue. La logique floue dit qu'un état ne peut être défini qu'en fonction de l'objet. Par exemple : $5^{\circ} \mathrm{C}$ est froid pour l'objet «beurre »; alors que $5^{\circ} \mathrm{C}$ est chaud pour la « glace». Tout n'est donc que question de sensation pour l'objet 
et de frontière entre deux états. Chaque senseur est défini par rapport à l'objet étudié. La certitude vient que l'objet répond à différents senseurs plus ou moins incertains. Et donc, les poids affectés aux capteurs dans la théorie de Dempster-Shafer vont dans ce sens pour proposer une valeur. Vous suivez?

Serge-Francis : Oui je connais cette théorie depuis longtemps, Marie me l'avait expliquée. C'est ce que nous utilisons pour nos senseurs.

Sylvie : Moi je ne te suis pas du tout.

Fabrice : Bref, cette valeur dépend de l'objet ou encore de l'individu comme vous voulez. Celui-ci espère donc y trouver ce qu'il cherche. Et que cherchet-il ?

Serge-Francis : Je ne sais pas.

Sylvie : Moi non plus.

Fabrice : Et bien il cherche le «A Quoi» quoi. L'individu lui il n'a qu'un objectif, c'est de s'en servir. Il se moque du «pourquoi» et du «comment» et même du « quoi ». C'est ça le monde moderne.

Serge-Francis : (un peu énervé) Si je comprends bien, c'est l'individualisme poussé à l'extrême qui débouche sur des inégalités de plus en plus flagrantes. C'est ça la «théorie du A QUOI », et nous sommes nous, vous et moi, en plein dedans et on aime ça non ?

Serge-Francis : (plus calme) J'ai 68 ans, encore 2 ans et je suis à la retraite sauf si le gouvernement repousse encore ....

Sylvie : Ouais, je comprends en gros la théorie du « à quoi » et j'ai l'impression de la vivre tous les jours. Mais je ne comprends pas bien encore le rapport avec nos activités...

Au fait, on m'a dit que les chercheurs du Labex sont en grève, ils désirent une prime de la connaissance ?

Fabrice : Oh eux, je ne vois ce qu'ils peuvent bien vouloir, ce sont quand même des fonctionnaires assez privilégiés...

Serge-Francis : (énervé) Ouais, c'est ça, et vous allez me dire encore qu'ils sont trop payés, qu'ils glandent. Et que maintenant avec l'Intelligence Artificielle et le A Quoi, comme vous dites, nous suffisent comme à la société...

Mais, heureusement qu'ils sont venus contrôler les divagations des senseurs du dernier robot livré aux Russes.

Fabrice : C'est ça, c'est ce que je dis, ils servent à quoi ? A contrôler c'est tout ...

Tu dis, dans combien de temps la retraite ?

Serge-Francis : Putain, j'en viens presque à regretter Marie... Je me barre.

Fabrice : Ou vas-tu ?

Serge-Francis : Je vais essayer de retrouver mon sens critique, ça fait trente ans que je l'ai perdu.

(Serge-Francis regarde son café avec un regard critique sur ce qu'il boit depuis 30 ans, il le jette en grimaçant puis il sort.)

\subsection{Scène 2 : Sylvie et Fabrice regardent partir Serge-Francis.}

Sylvie : Mais quelle mouche le pique, je ne l'ai jamais vu comme ça. Il tient des propos bizarres. Lui si prompt à savoir faire faire le travail aux autres !

Fabrice : Ouais, il est bizarre, ça lui passera. Tu viens c'est l'heure de retrouver les Russes.

Sylvie : Non pas tout de suite...

(Fabrice doute, regarde la poubelle où le café s'étend! Il part vers son bureau. Sylvie, étonnée, le regarde partir.)

\section{CONCLUSION :}

Prendre le «A QUOI» comme avenir est une projection non irréelle si nous nous référons à la société actuelle. Remplacer les capteurs par des senseurs est plus discutable, mais pourquoi pas! Assimiler la théorie du «A Quoi» avec la logique floue devient presque logique !

A vous de juger.... Dans le futur.

(à suivre... peut-être!)

\section{RETOUR DES ETUDIANTS:}

Cette pièce, sans prétention, pourrait rentrer dans le cadre de Théâtre et Sciences [9] [10] que j'anime avec Bernard AVRON depuis 1989 et depuis cette année avec Karine DUFAUT. Les objectifs de Théâtre et Sciences sont de faire écrire, mettre en scène et faire jouer des étudiants sur un thème scientifique. Dans leur spectacle souvent il y a transposition du thème sur leurs préoccupations (éducation, drogue, liberté, sexe, origine, cultures, folie ...). Il traite aussi du thème en libérant leur créativité et leur imaginaire sur le thème, c'est sans doute le cas du texte théâtral que je vous ai proposé.

Nous avons réalisé une enquête sur Théâtre et Sciences il y a quelques temps. Les résultats sont résumés ci-après :

Les jeunes, interrogés, apprécient que Théâtre et Sciences, fenêtre ouverte sur le monde extrauniversitaire, occupe un véritable espace théâtral, en centre ville. $100 \%$ se déclarent «très satisfaits » ou « satisfaits » de leur expérience.

Les apports, qu'ils estiment parfois se situer «au-delà de ce qui est exprimable », sont de deux ordres: culturel $(80 \%$ disent avoir acquis des connaissances importantes et variées) et surtout personnel, leurs effets se faisant sentir tant dans la relation à soi-même que dans la relation à autrui.

Les interviewés disent «avoir pu surmonter leur timidité », « avoir gagné de l'assurance, de la confiance en eux », «être plus à l'aise pour s'exprimer en public », « avoir développé leur imagination, ». Mieux encore, ils pensent se connaître mieux, avoir une 
personnalité, une force morale plus affirmée et être plus à même de s'adapter rapidement à des situations inconnues.

Etant mieux avec eux-mêmes, ils sont «plus ouverts aux autres », ont une «meilleure écoute », ils « savent mieux exprimer, extérioriser ce qu'ils ressentent », ils ont acquis «l'esprit d'équipe» et «le sens de la communication ».

Ce sont les réactions après 6 mois de répétition (4 heures par semaine et 2 week-ends) et quelques spectacles à Clermont-Ferrand ou ailleurs. J'ai aussi mis en place avec Bernard, des stages de 2 jours sur le même principe, les évaluations des étudiants qui ont suivi ces stages sont pratiquement identiques...

Ce sont presque les mêmes apports que je ressens en publiant ce texte, étonnant non !

\section{MES DEFINITIONS :}

A QUOI : C'est une constatation plus qu'une théorie. Il est clair que de plus en plus nous utilisons les objets sans connaître le pourquoi et le comment ni le quoi. A quoi ça sert? A quoi ça met utile? Sont les questions que nous nous posons! Vivons-nous dans une civilisation du A QUOI? Ces objets me donnent des sensations et j'aime ça! Je ne capte plus, je ressens.... Capteurs ou senseurs?

Bibi binaire : Nous comptons en base 10, pourquoi ?... Bobby Lapointe a inventé un autre base tirée de la base 16 ou hexadécimale $\left((2)^{2}\right)^{2}$ (bi puissance bi puissance bi) d'où son nom ! (binaire est le langage des ordinateurs, un assemblage de 0 et de 1). Les chiffres en bibi binaire sont: HO, HA, HE, HI, BO, BA, BE, BI, KO, KA, KE, KI, DO, DA, DE, DI. (0 à 15 en décimal).

Et : $D A * D O=K A D O$. C'est autrement plus joli que $(13 * 12=156$ en décimal $)$ ! ou que $\left(D^{*} C=8 C\right.$ en hexadécimal)!

Calibrer: Un capteur doit donner une mesure la plus juste possible à une erreur d'incertitude près, on dit aussi étalonner. Comme l'étalonnage de la balance chez votre boucher qui doit donner un poids juste ? Et, elle se règle par rapport à une référence (étalon). Le modèle de la balance est de type fléau, le réglage de l'équilibre est simple. Pour des capteurs plus compliqués (par exemple non linéaire comme un capteur de température), les modèles sont plus difficiles à appréhender. Ils sont souvent définis par une équation différentielle.

Capteurs actifs : Par rapport aux capteurs passifs, ils émettent une onde qui peut être perturbante voire dangereuse pour le milieu ou l'objet. Par exemple un télémètre laser qui mesure des distances ou encore des rayons $\mathrm{X}$ qui donnent une image radiologique (Attention ça brule...).

Capteurs passifs : Ils sont sensibles à une information particulière (onde lumineuse, gaz...) certains sont sans contact avec l'objet à détecter ou à mesurer (par exemple caméra).

Connaissances : Acquérir des connaissances est pris au sens des savoirs pour comprendre.

Compétences: Ici le sens est : J'apprends pour savoir faire.

Je fais la distinction entre connaissances et compétences; car fabriquer peut, certes, ne se faire qu'avec des compétences; mais il faut des connaissances pour évoluer. Bon ok, vous avez le droit de ne pas être d'accord! Jouer sur les mots ça permet de se poser des questions!

Equations différentielles: Elles sont utilisées pour construire des modèles mathématiques pour décrire (modéliser) des phénomènes physiques, biologiques ou autres (gravitation, circuits électriques, évolution de population...). Elles ont été développées par Leibniz (1686) et Newton.

Essais/erreurs: Méthode scientifique simple où en expérimentant on trouvera bien un jour une solution! Devise Shadok: «En essayant continuellement on finit par réussir. Donc: Plus ça rate, plus on a de chance que ça marche...»

Gestion des Ressources Humaines (GRH) : Dans l'histoire de l'exploitation de l'Homme par l'Homme, on connait l'esclavage, le servage, le Taylorisme, l'organisation scientifique du travail, maintenant la GRH depuis quelques années! Naturellement tout cohabite, vive le progrès!

Incertitudes: Une mesure n'est jamais juste mais associée à une erreur. Par exemple pour un thermomètre: KADO degrés à plus ou moins BA degrés. Elles peuvent être multiples et dues par exemple : à la constitution et à la fabrication d'un capteur (rapport Signal/bruit), au modèle utilisé pour l'étalonner, aux perturbations externes (ondes électromagnétiques...), à un biais systématique ...

Intelligence Artificielle (IA): Tout le monde sait ce qu'est l'IA ? Ce sont des acquisitions virtuelles de connaissances et de stratégies pour comprendre et agir (jeux, robotique ...). Mais l'intelligence humaine fait appel à la Pensée qui n'est pas électronique encore moins informatique, peut-être un jour biologique? Je Pense donc je Suis. Mais je peux Etre sans Penser ou sans Rêver!

LABEX : Laboratoires d'excellence ayant une visibilité internationale donc digne d'être financé... gare aux autres!

Logique floue (Lotfi ZADEH 1965): J'ai toujours adoré l'association de ces deux mots la logique de la rigueur et le flou artistique! En fait, le flou est toujours aux frontières. Est-ce chaud ou froid ? Estce imprécis, incertain, probable, possible ? Cette théorie est répandue en automatique, environnement (climat), aide au diagnostic, étude des risques...

Logique numérique: C'est la logique des ordinateurs (mais pas seulement) qui mêlent les 0 et les 1 
associés à une algèbre particulière dite de BOOLE (1815-1864).

MATLAB : C'est un logiciel proposant une boite à outils virtuelle et payante qui permet de résoudre beaucoup de problèmes mathématique, physique, signal, biologique ... Il est construit sur la base du calcul matriciel.

Modèle : Nous avons toutes et tous un modèle voire plusieurs, même les machines. Mais, un modèle reste un modèle plus ou moins éloigné de la réalité. Même s'il est top! Un modèle est une approximation de la réalité. Donc un top modèle est le top de l'approximation et non pas le top de la perfection comme on veut nous le faire croire!

Modéliser: C'est rechercher une méthode mathématique (équation différentielle par exemple) ou informatique (algorithmes) pour définir le clone virtuel d'un système réel. Ceci peut permettre de travailler virtuellement sur le système donc, sans risque pour le système réel. Mais aussi, et c'est le cas ici, de pouvoir le calibrer.

Moindres carrés: C'est une méthode pour pouvoir définir les paramètres d'un modèle par rapport à une référence réelle. On minimise l'écart au carré entre les mesures réelles et modèle. Pour cela on annule la dérivée de cet écart. Connaissant les paramètres du modèle, il est entièrement connu. Enfin on espère entièrement le connaître!

Relativité restreinte: Je m'interroge sur les relations entre espace et temps. La durée dépend de la vitesse, je vieillirai moins vite à grande vitesse? Néanmoins le temps passe plus vite sur tes genoux que sur un poêle. Nous tenons compte de cette théorie et de celle naturellement de la gravité pour positionner précisément un satellite dans l'espace.

Revue Nature : Revue généraliste de référence, une des plus réputée du monde...

Senseurs: Normalement synonymes de capteurs. Je fais une différence importante dans le texte car ils donnent des sensations et non pas des mesures.

Un capteur donne une mesure. Par exemple un carré de couleur bleu correspond à une longueur d'onde précise. Le capteur la mesure. Si ce carré est entouré de rouge, la mesure du bleu sera toujours la même. Pourtant pour nous la sensation de bleu ne sera pas la même. Je suis aussi sensible à l'environnement.

Le capteur donne une information pour souvent, en automatique, asservir un système. Nos informations nous asservissent-elles? Faisons plutôt confiance à nos sensations et à notre esprit critique...

Sonar : Capteur actif qui émet des ondes sonores pour, par exemple, analyser les fonds marins.

Télémètre : Capteur actif qui mesure des distances. Il envoie une onde (souvent une onde laser) qui réfléchit sur l'objet revient sur le capteur. Il suffit de calculer la durée entre émission et réception de l'onde pour avoir une mesure de la distance directement proportionnelle à cette durée.
Théorie de Dempster-Shafer: Théorie mathématique (1976) qui, à partir de données distinctes, permet de calculer la probabilité qu'un évènement se produise.

Théorie de l'évidence: Techniques de fusion de données incertaines. Complète la théorie de Dempster-Shafer. C'est évidemment incertain!

UER : Unité d'Enseignement et de Recherche pour une discipline (sciences, lettres ...). C'était le nom de ces unités avant la loi Savary (1984).

UFR : Unité de Formation et Recherche, nom donné après la loi Savary. Changer «enseignement» en «formation » n'est pas sans conséquence. Former, Formater ou Déformer ou Déformater!

$A h$, vous trouvez encore que je joue trop sur les mots!

\section{Bibliographie :}

[1] Hans BAUER, «Atlas de la Physique », Livre de poche collection: Encyclopédies d'aujourd'hui, 1997.

[2] Temps, matière et espace Hors série science et vie septembre 2012.

[3] Bernadette BOUCHON-MEUNIER, «La logique floue », Presses universitaires de France, collection : "Que sais-je » 2007.

[4] Didier DUBOIS, Henri PRADE. «Théorie des Possibilités. Applications à la Représentation des Connaissances en Informatique », Collection Méthode + Programmes, Masson, Paris, 1985.

[5] Dorothée BRUNET-LECOMTE, «Au son du sonar, la vie de la baleine vire au cauchemar », Libération, 9 octobre 2003.

[6] Juan José ALAVA, «Cetaceans: Monitor sea pollution to stop strandings », journal Nature 486, 323, 21 juin 2012.

[7] Bobby LAPOINTE, «Le langage bibi binaire » 1968

http://www.ac-grenoble.fr/college/pont-declaix.moucherotte/pedagogi/maths/mathamus/bobb y-lap/bobbylap.htm.

[8] François FABRE, «Si le bibi de Bobby m'était compté » 2008,

http://www.billetreduc.com/66609/evt.htm

[9] Pierre BONTON, Bernard AVRON, «Un atelier «THEATRE et SCIENCES» pour une autre vision. » CETSIS 2003, Toulouse.

[10] Pierre BONTON «Les salles de créativité scientifique et technologique font-elles appel au sens du bricolage des étudiants? Application en électronique et au théâtre et sciences », colloque d'ESCHIL, 27 et 28 novembre 2008, "Génie(s) de la bricole et du bricolage », à l'INSA de Lyon.

PS:

Sylvie : A QUOI ça sert tous ces blablas?

Professeur ZALEIG : A rien, c'est justement dans ces moments de rien que je pense! Que je rêve... 